\title{
On Wieand's theorem
}

\author{
Wilbert C.M. Kallenberg ${ }^{\text {a }}$, Alex J. Koning ${ }^{\text {b,* }}$ \\ a Department of Applied Mathematics, University of Twente, P.O. Box 217, NL-7500 AE Enschede, The Netherlands \\ ${ }^{\mathrm{b}}$ Econometric Institute, Erasmus University, P.O. Box 1738, NL-3000 DR Rotterdam. The Netherlands
}

Received February 1994; revised September 1994

\begin{abstract}
Wieand's theorem on equivalence of limiting approximate Bahadur efficiency and limiting Pitman efficiency is extended in several ways. Conditions on monotonicity and continuity are obviated, composite null hypotheses are incorporated, and the implications of a weaker form of Wieand's Condition III* are investigated.
\end{abstract}

AMS 1991 Subject Classification: 62F05, 62G20

Keywords: Approximate Bahadur efficiency; Pitman efficiency

\section{Introduction}

To compare the performance of two sequences of test statistics, many efficiency concepts have been proposed. Probably the most widely used is asymptotic relative Pitman efficiency.

In Bahadur (1960) the concept of exact Bahadur efficiency was proposed. This concept requires large deviation results, the derivation of which often becomes the stumbling-block in the application. As a "quick and dirty" variant Bahadur simultaneously proposed approximate Bahadur efficiency, valid for comparison of the so-called standard sequences. Since it is rather easy to verify whether a sequence of test statistics actually is standard, approximate Bahadur efficiency in spite of its apparent shortcomings (cf. Bahadur, 1960) has become quite popular.

In favour of approximate Bahadur efficiency, Bahadur argued that for many well-known test statistics the limiting (as the alternative approaches the null hypothesis) approximate Bahadur efficiency is equal to the asymptotic relative Pitman efficiency. Working with an extended version of asymptotic relative Pitman efficiency, Wieand (1976) elaborated this point by presenting a condition under which the limiting approximate Bahadur efficiency coincided with the limiting (as the size of the test tends to zero) asymptotic relative Pitman efficiency.

Unfortunately, Wieand only proved the theorem which stated this coincidence for the simple null hypothesis. Moreover, he requires continuity and strict monotonicity of the tail of the asymptotic null distribution of the

* Corresponding author. 


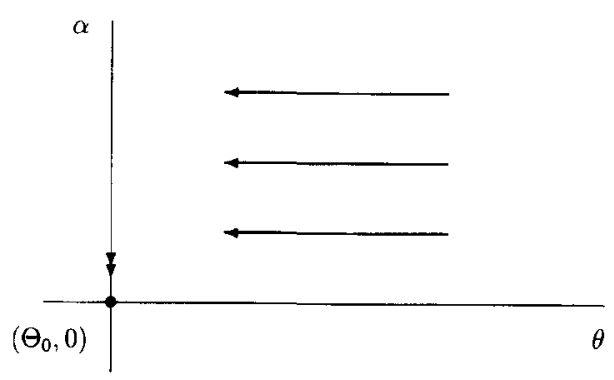

Fig. 1. Limiting Pitman efficiency.

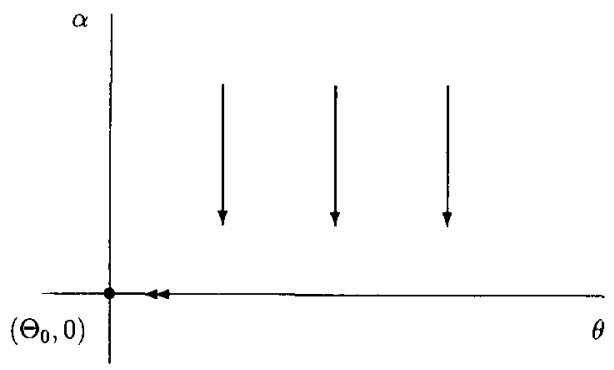

Fig. 2. Limiting Bahadur efficiency.

sequence of test statistics. In this note we extend Wieand's theorem to composite null hypotheses, while discarding continuity and strict monotonicity conditions.

Evaluating limiting Pitman efficiency means that the alternative is sent to the null hypothesis and afterwards the size $\alpha$ to zero. This is outlined in Fig. 1 .

The order of the operations is reversed for the limiting approximate Bahadur efficiency, as is sketched in Fig. 2.

Under Wieand's Condition III* it holds for standard sequences of test statistics that both ways of approaching $\left(\Theta_{0}, 0\right)$ yield the same result. In fact it can be shown that for many ways in which $(\theta, \alpha)$ tends to $\left(\Theta_{0}, 0\right)$ the result is the same, provided that Wieand's Condition III* holds.

Often, the knowledge of the behavior of the test statistics is available in great detail under the null hypothesis, but scarse under the alternative hypothesis. This may lead to problems with the verification of Wieand's Condition III*, since it deals explicitly with the behavior under the alternative hypothesis. However, if a weaker form of the condition holds (cf. Definition 3 in Section 2) it still can be shown that we come close to the answer of the Bahadur approach for any trajectory in a restricted region, for instance trajectories as sketched in Fig. 3.

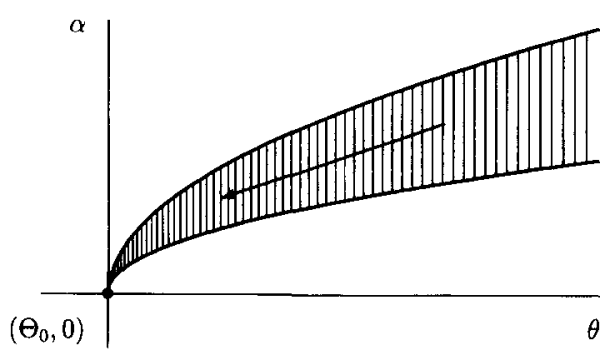

Fig. 3. The restircted area of admitted trajectories. 
The closer we want to be to the answer of the Bahadur approach, the smaller the area of admitted trajectories. This is, e.g. reflected by a lowering of the upper curve depicted in Fig. 3, and implies that we should restrict ourselves to tests of even smaller size.

Thus, the quality of the approximation of the finite sample relative efficiency by the approximate Bahadur efficiency for alternatives close to the null hypothesis is only guaranteed in case of (very) small levels.

The organization of the paper is as follows. In Section 2 notation is introduced and definitions are given. Section 3 contains the results, and Section 4 some examples. Proofs are gathered in Section 5.

\section{Preliminaries}

Consider the situation in which we have two infinite sequences of test statistics $\left\{T_{1 n}\right\}_{n=1}^{\infty}$ and $\left\{T_{2 n}\right\}_{n=1}^{\infty}$, rejecting the null hypothesis $H_{0}: \theta \in \Theta_{0}$ for large values of the test statistics. The number $n$ here refers to the number of available observations. The whole parameter space is denoted by $\Theta$, which is a metric space with metric $d$. For each $\alpha \in(0,1)$ the critical value is denoted by $t_{i n}(\alpha)$. So, $H_{0}$ is rejected if $T_{\text {in }}>t_{\text {in }}(\alpha)$, accepted if $T_{i n}<t_{i n}(\alpha)$, and

$$
\sup _{\theta_{0} \in \Theta_{0}} P_{\theta_{0}}\left(T_{\text {in }}>t_{\text {in }}(\alpha)\right) \leqslant \alpha \leqslant \sup _{\theta_{0} \in \Theta_{0}} P_{\theta_{0}}\left(T_{\text {in }} \geqslant t_{\text {in }}(\alpha)\right) \text {. }
$$

Definition 1. For each $\alpha, \beta \in(0,1)$ and $\theta \in \Theta-\Theta_{0}$, let $N_{i}(\alpha, \beta, \theta)$ be the largest sample size such that the power at $\theta$ of the size $\alpha$ test based on $\left\{T_{i n}\right\}_{n=1}^{\infty}$ is less than $\beta$.

The finite sample relative efficiency of $\left\{T_{1 n}\right\}_{n=1}^{\infty}$ with respect to $\left\{T_{2 n}\right\}_{n=1}^{\infty}$ is denoted by $\mathrm{N}_{2}(\alpha, \beta, \theta) / \mathrm{N}_{1}(\alpha, \beta, \theta)$. A value larger than 1 indicates that $\left\{T_{1 n}\right\}_{n=1}^{\infty}$ is preferred to $\left\{T_{2 n}\right\}_{n=1}^{\infty}$, since $\left\{T_{1 n}\right\}_{n=1}^{\infty}$ needs less observations for the same performance.

Definition 2. $\left\{T_{i n}\right\}_{n=1}^{\infty}$ is said to be a standard sequence if the following three conditions are satisfied.

(a) There exists a nondecreasing function $G_{i}$ such that $\inf _{\theta_{0} \in \Theta_{0}} P_{\theta_{0}}\left(T_{i n} \leqslant t\right)$ tends to $G_{i}(t)$ for all continuity points of $G_{i}$.

(b) There exists a constant $a_{i}>0$ such that

$$
\lim _{t \rightarrow \infty} t^{-2} \log \left(1-G_{i}(t)\right)=-a_{i} / 2 .
$$

(c) There exists a positive function $b_{i}(\theta)$ such that $\left|n^{-1 / 2} T_{i n}-b_{i}(\theta)\right|$ converges to zero in $P_{\theta}$-probability for every $\theta \in \Theta-\Theta_{0}$.

The approximate Bahadur slope of a standard sequence $\left\{T_{i n}\right\}_{n=1}^{\infty}$ is defined as $a_{i}\left(b_{i}(\theta)\right)^{2}$. The approximate Bahadur efficiency of a standard sequence $\left\{T_{1 n}\right\}_{n=1}^{\infty}$ with respect to another standard sequence $\left\{T_{2 n}\right\}_{n=1}^{\infty}$ is defined as the ratio of their respective Bahadur slopes $a_{1}\left(b_{1}(\theta)\right)^{2} /\left[a_{2}\left(b_{2}(\theta)\right)^{2}\right]$.

This is a slightly weaker form of the definition of standard sequences given in Bahadur (1960), not requiring continuity of $G_{i}$.

For a standard sequence $\left\{T_{i n}\right\}_{n=1}^{\infty}$ define for each $0<\alpha<1$

$$
\underline{q}_{i}(\alpha)=\inf \left\{t: 1-G_{i}(t) \leqslant \alpha\right\}, \bar{q}_{i}(\alpha)=\sup \left\{t: 1-G_{i}(t) \geqslant \alpha\right\} .
$$

By this definition and the monotonicity of $G_{i}$ we obtain

$$
\begin{array}{llll}
1-G_{i}(t)>\alpha & \text { for all } t<\underline{q}_{i}(\alpha), & 1-G_{i}(t) \geqslant \alpha & \text { for all } t<\bar{q}_{i}(\alpha), \\
1-G_{i}(t) \leqslant \alpha & \text { for all } t>\underline{q}_{i}(\alpha), & 1-G_{i}(t)<\alpha & \text { for all } t>\bar{q}_{i}(\alpha) .
\end{array}
$$


It is easily seen that

$$
\underline{q}_{i}(\alpha) \leqslant \bar{q}_{i}(\alpha)
$$

In view of Definition 2(a), denote for each $\varepsilon>0$ and $0<\alpha<1$ by $\bar{n}_{i}(\alpha, \varepsilon)$ the smallest number such that for all $n \geqslant \bar{n}_{i}(\alpha, \varepsilon)$

$$
1-\inf _{\theta_{0} \in \Theta_{0}} P_{\theta_{0}}\left(T_{i n} \leqslant \bar{q}_{i}(\alpha)(1+\varepsilon)^{1 / 2}\right)<\alpha .
$$

Similarly denote for each $\varepsilon>0$ and $0<\alpha<1$ by $\underline{n}_{i}(\alpha, \varepsilon)$ the smallest number such that for all $n \geqslant \underline{n}_{i}(\alpha, \varepsilon)$

$$
1-\inf _{\theta_{0} \in \Theta_{0}} P_{\theta_{0}}\left(T_{i n}<\underline{q}_{i}(\alpha)(1-\varepsilon)^{1 / 2}\right)>\alpha .
$$

Define $n_{i}(\alpha, \varepsilon)$ by

$$
n_{i}(\alpha, \varepsilon)=\max \left(\bar{n}_{i}(\alpha, \varepsilon), \underline{n}_{i}(\alpha, \varepsilon)\right) .
$$

Finally we present the weaker form of Wieand's Condition III*. $^{*}$.

Definition 3. A standard sequence $\left\{T_{i n}\right\}_{n=1}^{\infty}$ is said to be a $\left\{s_{n}\right\}_{n=1}^{\infty}$-Wieand sequence if there is a constant $\varepsilon_{i}^{*}>0$ such that for every $\varepsilon>0$ and $\delta \in(0,1)$ there exists a constant $C_{i}(\varepsilon, \delta)$ such that

$$
P_{\theta}\left(\left|n^{-1 / 2} T_{\text {in }}-b_{i}(\theta)\right| \geqslant \varepsilon b_{i}(\theta)\right)<\delta
$$

for every $\theta \in \Theta-\Theta_{0}$ and $n \in \mathbb{R}$ satisfying $\inf _{\theta_{0} \in \Theta_{0}} d\left(\theta, \theta_{0}\right)<\varepsilon_{i}^{*}$ and $n^{1 / 2} b_{i}(\theta)>C_{i}(\varepsilon, \delta) s_{n}$.

It can be shown that a standard sequence $\left\{T_{i n}\right\}_{n=1}^{\infty}$ is a $\left\{s_{n}\right\}_{n=1}^{\infty}$-Wieand sequence if there exists a nondefective cumulative distribution function $Q$ such that

$$
P_{\theta}\left(\left|T_{i n}-n^{1 / 2} b_{i}(\theta)\right|>x s_{n}\right) \leqslant 1-Q(x)
$$

for every $x>0$ and $\theta \in \Theta-\Theta_{0}$ satisfying $\inf _{\theta_{0} \in \Theta_{0}} d\left(\theta, \theta_{0}\right)<\varepsilon_{i}^{*}$ [choose $C_{i}(\varepsilon, \delta)$ so as to satisfy $Q\left(\varepsilon C_{i}(\varepsilon, \delta)\right)>$ $1-\delta$ ]. Remark that if $s_{n}$ remains bounded, Definition 3 reduces to Wieand's Condition III* (in case of a simple null hypothesis), and the observation above is a consequence of the lemma given in Section 4 in Wieand (1976). In the sequel we shall use the phrase "under Wieand's Condition III" to indicate that $s_{n}$ remains bounded, thereby tacitly extending Wieand's original definition to general null hypotheses.

We close this section by introducing a set which plays a role in Section 3. For each $\alpha, \varepsilon, \delta \in(0,1)$ let $A(\alpha, \varepsilon, \delta)$ be the set of points $\theta \in \Theta-\Theta_{0}$ for which

$$
n \geqslant \frac{-\log \alpha}{a_{i}\left\{b_{i}(\theta)\right\}^{2}} \text { implies } n^{1 / 2} b_{i}(\theta)>C_{i}(\varepsilon, \delta) s_{n}
$$

for $i=1,2$. Lemma 1 sheds light on the relation between the set $A(\alpha, \varepsilon, \delta)$ and Wieand's Condition II $^{*}$. Observe that in case we have

$$
\inf _{\theta \in \theta-\theta_{0}} b_{i}(\theta)=0 \text { and } s_{n} \text { monotone increasing, }
$$

this lemma implies that $A(\alpha, \varepsilon, \delta)$ equals $\Theta-\Theta_{0}$ for $\alpha$ sufficiently small if and only if $s_{n}$ is bounded.

Lemma 1. If $s_{n}$ remains bounded then for every $\varepsilon, \delta>0$ there exists $\alpha(\varepsilon, \delta)>0$ such that $A(\alpha, \varepsilon, \delta)$ coincides with $\Theta-\Theta_{0}$ for every $0<\alpha<\alpha(\varepsilon, \delta)$. If $\inf _{\theta \in A(\alpha, \varepsilon, \delta)} b_{i}(\theta)=0$ for some $\alpha, \varepsilon, \delta>0$, then $\liminf _{n \rightarrow \infty} s_{n}<\infty$. 


\section{Results}

Our main result is the following theorem, which shows where the finite sample relative efficiency $\mathrm{N}_{2}(\alpha, \beta, \theta)$ / $\mathrm{N}_{1}(\alpha, \beta, \theta)$ may be approximated by the approximate Bahadur efficiency $a_{1}\left\{b_{1}(\theta)\right\}^{2} /\left[a_{2}\left\{b_{2}(\theta)\right\}^{2}\right]$. (If limits are taken with $\theta$ tending to some subset of $\Theta_{0}$, then of course $\theta$ runs through $\Theta-\Theta_{0}$; further the notation is explained in Section 2.)

Theorem 1. Let $\left\{T_{1 n}\right\}_{n=1}^{\infty}$ and $\left\{T_{2 n}\right\}_{n=1}^{\infty}$ be two $\left\{s_{n}\right\}_{n=1}^{\infty}$-Wieand sequences, and let $\Theta_{0}^{*}$ be a subset of $\Theta_{0}$. Suppose that the limit

$$
\lim _{\theta \rightarrow \Theta_{0}^{*}} a_{1}\left\{b_{1}(\theta)\right\}^{2} /\left[a_{2}\left\{b_{2}(\theta)\right\}^{2}\right]=: e\left(\Theta_{0}^{*}\right)
$$

exists. For each $\alpha, \varepsilon, \delta \in(0,1)$, let $A^{*}(\alpha, \varepsilon, \delta)$ be a subset of $A(\alpha, \varepsilon, \delta)$ satisfying

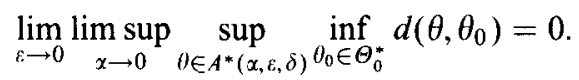

\section{Assume that}

$$
\limsup _{\varepsilon \rightarrow 0} \limsup _{\alpha \rightarrow 0} \sup _{\theta \in A^{*}(\alpha, \varepsilon, \delta)} \frac{n_{i}(\alpha, \varepsilon)}{\left[\frac{-2 \log \alpha}{a_{i}\left\{b_{i}(\theta)\right\}^{2}}\right]}<1 .
$$

Then for $\beta \in[\delta, 1-\delta]$ we have

$$
\lim _{\varepsilon \rightarrow 0} \liminf _{\alpha \rightarrow 0} \inf _{\theta \in A^{*}(\alpha, E, \delta)} \frac{\mathrm{N}_{2}(\alpha, \beta, \theta)}{\mathrm{N}_{1}(\alpha, \beta, \theta)}=\lim _{\varepsilon \rightarrow 0} \limsup _{\alpha \rightarrow 0} \sup _{\theta \in A^{*}(\alpha, \varepsilon, \delta)} \frac{\mathrm{N}_{2}(\alpha, \beta, \theta)}{\mathrm{N}_{1}(\alpha, \beta, \theta)}=e\left(\Theta_{0}^{*}\right) .
$$

When considering a specified trajectory $\theta(\alpha, \varepsilon, \delta)$, along which $\Theta_{0}^{*}$ is approached as $\alpha \rightarrow 0$, it seems obvious to choose $A^{*}(\alpha, \varepsilon, \delta)$ equal to the singleton containing $\theta(\alpha, \varepsilon, \delta)$.

If Wieand's Condition III* does not hold, the requirement that $\theta(\alpha, \varepsilon, \delta)$ should be an element of $A(\alpha, \varepsilon, \delta)$ limits the trajectories admitted for a fixed $\varepsilon>0$. The lower $\varepsilon$, the smaller the area through which the trajectories are allowed to run. As already observed in Section 2, this is reflected in Fig. 3 by a lowering of the upper curve. For instance, if $n^{1 / 2} / s_{n}$ is a monotone increasing function $g$ of $n$, condition (10) holds if and only if

$$
g\left(\frac{-\log \alpha}{a_{i}\left\{b_{i}(\theta)\right\}^{2}}\right) b_{i}(\theta)>C_{i}(\varepsilon, \delta)
$$

for $i=1,2$. Moreover, if $s_{n}=\mathcal{O}\left(n^{\rho}\right)$, then there exist a positive constant $C_{i}^{*}(\varepsilon, \delta)$ such that

$$
\left\{\theta \in \Theta-\Theta_{0}: C_{i}^{*}(\varepsilon, \delta) \leqslant\left(\frac{-\log \alpha}{a_{i}}\right)^{1 / 2-\rho}\left\{b_{i}(\theta)\right\}^{2 \rho}\right\} \subset A(\alpha, \varepsilon, \delta)
$$

for all $\alpha, \varepsilon, \delta \in(0,1)$. The proof of (15) is in Section 5 .

If Wieand's Condition III $^{*}$ does hold, the set $A(\alpha, \varepsilon, \delta)$ equals $\Theta-\Theta_{0}$ if $0<\alpha<\alpha(\varepsilon, \delta)$, where $\alpha(\varepsilon, \delta)$ is given by Lemma 1. It now easily follows that for every sequence $\left\{\left(\theta_{j}, \alpha_{j}\right)\right\}_{j=1}^{\infty}$ tending to $\left(\Theta_{0}^{*}, 0\right)$ we obtain

$$
\frac{\mathrm{N}_{2}\left(\alpha_{j}, \beta, \theta_{j}\right)}{\mathrm{N}_{1}\left(\alpha_{j}, \beta, \theta_{j}\right)} \rightarrow e\left(\Theta_{0}^{*}\right)
$$


provided that

$$
\limsup _{\varepsilon \rightarrow 0} \lim _{j \rightarrow \infty} \frac{n_{i}\left(\alpha_{j}, \varepsilon\right)}{\left[\frac{-2 \log \alpha_{j}}{a_{i}\left\{b_{i}\left(\theta_{j}\right)\right\}^{2}}\right]}<1,
$$

as is formally seen by taking $A^{*}(\alpha, \varepsilon, \delta)$ equal to $\left\{\theta_{j}\right\}$ if $\alpha_{j} \leqslant \alpha<\alpha_{j-1}, j=1,2, \ldots\left[\alpha_{0}=1\right]$. This is closely related to intermediate efficiency (cf. Kallenberg, 1983).

Another consequence of Lemma 1 is that under Wieand's Condition $\operatorname{III}^{*}$ we may take $A^{*}(\alpha, \varepsilon, \delta)$ to be the intersection of $\Theta-\Theta_{0}$ with an arbitrary environment of $\Theta_{0}^{*}$, shrinking to $\Theta_{0}^{*}$ as $\alpha \rightarrow 0$ and (afterwards) $\varepsilon \rightarrow 0$. More precisely, let

$$
A^{*}(\alpha, \varepsilon, \delta)=\left\{\theta \in A(\alpha, \varepsilon, \delta): \inf _{\theta_{0} \in \Theta_{0}^{*}} d\left(\theta, \theta_{0}\right)<\varepsilon, \frac{n_{i}(\alpha, \varepsilon)}{1+\varepsilon}<\frac{-2 \log \alpha}{a_{i}\left\{b_{i}(\theta)\right\}^{2}}\right\} .
$$

Then (12) and (13) are satisfied. Now consider a sequence $\left\{\theta_{j}\right\}_{j=1}^{\infty}$ which tends to $\Theta_{0}^{*}$ as $j \rightarrow \infty$. Assume that

$$
\lim _{j \rightarrow \infty} b_{i}\left(\theta_{j}\right)=0
$$

Let $\alpha, \varepsilon, \delta>0$ satisfy $\alpha<\alpha(\varepsilon, \delta)$, with $\alpha(\varepsilon, \delta)$ as in Lemma 1 . Then there exists $j(\alpha, \varepsilon, \delta)$ such that for all $j \geqslant j(\alpha, \varepsilon, \delta)$ we have $\theta_{j} \in A^{*}(\alpha, \varepsilon, \delta)$, and hence it follows that

$$
\inf _{\theta \in A^{*}(\alpha, \varepsilon, \delta)} \frac{\mathrm{N}_{2}(\alpha, \beta, \theta)}{\mathrm{N}_{1}(\alpha, \beta, \theta)} \leqslant \liminf _{j \rightarrow \infty} \frac{\mathrm{N}_{2}\left(\alpha, \beta, \theta_{j}\right)}{\mathrm{N}_{1}\left(\alpha, \beta, \theta_{j}\right)} \leqslant \limsup _{j \rightarrow \infty} \frac{\mathrm{N}_{2}\left(\alpha, \beta, \theta_{j}\right)}{\mathrm{N}_{1}\left(\alpha, \beta, \theta_{j}\right)} \leqslant \sup _{\theta \in A^{*}(\alpha, \varepsilon, \delta)} \frac{\mathrm{N}_{2}(\alpha, \beta, \theta)}{\mathrm{N}_{1}(\alpha, \beta, \theta)} .
$$

Applying Theorem 1 we get

$$
\begin{aligned}
e\left(\Theta_{0}^{*}\right) & =\lim _{\varepsilon \rightarrow 0} \liminf _{\alpha \rightarrow 0} \inf _{\theta \in A^{*}(\alpha, \varepsilon, \delta)} \frac{\mathrm{N}_{2}(\alpha, \beta, \theta)}{\mathrm{N}_{1}(\alpha, \beta, \theta)} \\
& \leqslant \liminf _{\alpha \rightarrow 0} \liminf _{j \rightarrow \infty} \frac{\mathrm{N}_{2}\left(\alpha, \beta, \theta_{j}\right)}{\mathrm{N}_{1}\left(\alpha, \beta, \theta_{j}\right)} \leqslant \limsup \limsup _{\alpha \rightarrow 0} \frac{\mathrm{N}_{2}\left(\alpha, \beta, \theta_{j}\right)}{\mathrm{N}_{1}\left(\alpha, \beta, \theta_{j}\right)} \\
& \leqslant \lim _{\varepsilon \rightarrow 0} \limsup _{\alpha \rightarrow 0} \sup _{\theta \in A^{*}(\alpha, \varepsilon, \delta)} \frac{\mathrm{N}_{2}(\alpha, \beta, \theta)}{\mathrm{N}_{1}(\alpha, \beta, \theta)}=e\left(\Theta_{0}^{*}\right)
\end{aligned}
$$

and therefore

$$
\lim _{\alpha \rightarrow 0} \lim _{j \rightarrow \infty} \frac{\mathrm{N}_{2}\left(\alpha, \beta, \theta_{j}\right)}{\mathrm{N}_{1}\left(\alpha, \beta, \theta_{j}\right)}=e\left(\Theta_{0}^{*}\right) .
$$

Since the sequence $\left\{\theta_{j}\right\}_{j=1}^{\infty}$ was arbitrarily chosen, it follows that

$$
\lim _{x \rightarrow 0} \lim _{\theta \rightarrow \Theta_{0}^{*}} \frac{\mathrm{N}_{2}(\alpha, \beta, \theta)}{\mathrm{N}_{1}(\alpha, \beta, \theta)}=e\left(\Theta_{0}^{*}\right) .
$$

This extends Wieand's theorem to testing problems with composite null hypotheses. Moreover, note that Wieand's conditions on continuity and monotonicity are not needed to obtain this result.

There exist alternative definitions of limiting Pitman efficiency which involve $\underline{N}_{i}(\alpha, \beta, \theta)$, the smallest sample size such that the power at $\theta$ of the size $\alpha$ test based on $\left\{T_{i n}\right\}_{n=1}^{\infty}$ is greater than or equal to $\beta$. Observe that there is a disproportionate effect of the behavior of $T_{i 1}$ on $\underline{\mathrm{N}}_{i}(\alpha, \beta, \theta)$. Definition 3 only has meaning for sample sizes which are sufficiently large, and is therefore not capable of providing lower bounds for $\underline{N}_{i}(\alpha, \beta, \theta)$. It 
follows that the fact that $\left\{T_{i n}\right\}_{n=1}^{\infty}$ is a $\left\{s_{n}\right\}_{n=1}^{\infty}$-Wieand sequence does not shed light on asymptotic relative Pitman efficiency as defined in for instance Rothe (1981).

In absence of Wieand's Condition III $^{*}$ the verification of (13) requires some effort. In case the test statistics are functionals of either the empirical process or the partial sum process, the approximation theorems available for these processes (cf. Komloś et al., 1975) may lead to probability inequalities of the type considered in the following lemma.

Lemma 2. Suppose that $\left\{T_{i n}\right\}_{n=1}^{\infty}$ is a standard sequence for which there exist a random variable $T_{i}$ with distribution function $G_{i}$, constants $\kappa \geqslant \frac{1}{2}, \gamma>0, \lambda>0$ and a sequence $k_{n}=\mathcal{O}\left(n^{\gamma / \kappa}\right)$ such that

$$
\sup _{0_{0} \in \Theta_{0}} P_{\theta_{0}}\left(\left|T_{i n}-T_{i}\right|>n^{-\gamma}\left(k_{n}+x\right)^{k}\right) \leqslant \mathrm{e}^{-i x} .
$$

Then for every $\varepsilon>0$ there exist a constant $c(\varepsilon)$ such that (13) holds for

$$
A^{*}(\alpha, \varepsilon, \delta)=\left\{\theta \in A(\alpha, \varepsilon, \delta): a_{i}\left\{b_{i}(\theta)\right\}^{2}<c(\varepsilon)|\log \alpha|^{1-(\kappa-1 / 2) / \%}\right\}
$$

Typically, one finds that (18) is satisfied with $\gamma \leqslant \frac{1}{2}, \kappa \geqslant 1$ and $k_{n}$ proportional to $\log n$ (cf. Komlós et al., 1975; Csörgő and Horváth, 1993; Koning, 1992; 1994a, b).

The magnitude of $k_{n}$ allowed in Lemma 2 strongly suggests that it is essentially the tail rather than the center of the distribution of $n^{\gamma}\left|T_{i n}-T_{i}\right|$ which determines the shape of $A^{*}(\alpha, \varepsilon, \delta)$.

Lemma 2 in Koning (1994a) shows that (2) and (18) with $k_{n}$ proportional to $\log n$ together imply the deviation result

$$
\lim _{n \rightarrow \infty}\left(x_{n}\right)^{-2} \log P\left(T_{\text {in }}>x_{n}\right)=-a_{i} / 2
$$

for sequences $\left\{x_{n}\right\}_{n=1}^{\infty}$ such that $x_{n} \rightarrow \infty$ and $x_{n}=o\left(n^{\gamma /(2 \kappa-1)}\right)$. Deviation results have relevance for intermediate efficiency (cf. Kallenberg, 1983).

\section{Examples}

Let $X_{1}, \ldots, X_{n}$ be independent non-negative random variables, having common continuous cumulative distribution function $F(\cdot ; \theta)$ under $P_{\theta}$, and denote $-\log (1-F(x ; \theta))$ by $A(x ; \theta)$. Assume the existence of constants $v \in(0,1)$ and $c_{v}<\infty$ such that

$$
\int_{[0, t]}(1-F(s ; \theta))^{v} \mathrm{~d} \Lambda\left(s ; \theta_{0}\right)<c_{v} \quad \text { for every } \theta \in \Theta .
$$

The constant $v$ indicates the impact the alternatives have on the tail of the distribution of the $X_{i}$ 's. We shall distinguish between moderate tail alternatives $0<v<\frac{1}{2}$ and heavy tail alternatives $\frac{1}{2}<v<1$.

Define the empirical process by

$$
U_{n}(t ; \theta)=\frac{1}{n^{1 / 2}} \sum_{i=1}^{n}\left\{1_{\left\{X_{i} \leqslant t\right\}}-F(t ; \theta)\right\}
$$

and consider the sequence of test statistics $T_{\text {in }}$ defined by

$$
T_{i n}=\sup _{t \in[0, \infty)} \int_{[0, t]} \frac{U_{n}\left(s-; \theta_{0}\right)}{1-F\left(s ; \theta_{0}\right)} \mathrm{d} F\left(s ; \theta_{0}\right)=\sup _{t \in[0, \infty)} \int_{[0 . t]} U_{n}\left(s-; \theta_{0}\right) \mathrm{d} A\left(s ; \theta_{0}\right) .
$$


It is easily seen that we may write

$$
\left|T_{\text {in }}-n^{1 / 2} b_{i}(\theta)\right| \leqslant c_{v} \sup _{t \in[0, \infty)} \frac{\left|U_{n}(t ; \theta)\right|}{(1-F(t ; \theta))^{v}},
$$

where

$$
b_{i}(\theta)=\sup _{t \in[0, \infty)} \int_{[0, t]}\left(F(s ; \theta)-F\left(s ; \theta_{0}\right)\right) \mathrm{d} \Lambda\left(s ; \theta_{0}\right)
$$

(the existence of $b_{i}(\theta)$ is guaranteed by (19)). Hence, (4.14) in Marcus and Zinn (1984) yields

$$
P_{\theta}\left(\left|T_{\text {in }}-n^{1 / 2} b_{i}(\theta)\right|>x n^{v-1 / 2}\right) \leqslant \frac{257}{2 v-1}\left(\frac{3 c_{v}}{x}\right)^{1 / v}
$$

for heavy tail alternatives, and Lemma 2.2 in Pyke and Shorack (1968) yields

$$
P_{\theta}\left(\left|T_{i n}-n^{1 / 2} b_{i}(\theta)\right|>x\right) \leqslant \frac{1}{1-2 v}\left(\frac{c_{v}}{x}\right)^{2}
$$

for moderate tail alternatives. Observe that both probability inequalities are of the form (9). For moderate tail alternatives $\left\{T_{i n}\right\}_{n=1}^{\infty}$ is an ordinary Wieand sequence, but for heavy tail alternatives $\left\{T_{i n}\right\}_{n=1}^{\infty}$ is only a $\left\{n^{v-1 / 2}\right\}_{n=1}^{\infty}$-Wieand sequence.

Rather than exploring the test statistic (20) further, we now turn to the general class of test statistics for the simple null hypothesis in the random censoring model studied in Koning $(1992,1994 \mathrm{~b})$, which contains test statistics proposed in Breslow (1975), Aki (1986), Harrington and Fleming (1982) and the KolmogorovSmirnov type statistics considered in Section V.4.1 in Andersen et al. (1993) as special cases. In this class, say $\mathscr{K}$, the same distinction between moderate and heavy tail alternatives can be made.

In Koning (1992) it is shown that Wieand's Condition III* holds within $\mathscr{K}$ with respect to moderate tail alternatives (compare (19) with Condition 1 in Koning, 1992). One may show along similar lines that sequences of test statistics in $\mathscr{K}$ are $\left\{n^{v-1 / 2}\right\}_{n=1}^{\infty}$-Wieand sequences with respect to heavy tail alternatives. It is also shown in Koning (1992) that (18) is satisfied within $\mathscr{K}$ with $\kappa=2, \gamma=\frac{1}{6}, \lambda>0$ and $k_{n}=\log n$, and hence Lemma 2 applies. Moreover, (15) with $v-\frac{1}{2}<\rho<\frac{1}{18}$ yields that the set $A^{*}(\alpha, \varepsilon, \delta)$ introduced in Lemma 2 is non-empty. Thus, Theorem 1 gives an approximation for the finite sample relative efficiency in case the alternatives are heavily tailed with $\frac{1}{2}<v<\frac{5}{9}$.

The extension of the class $\mathscr{K}$ to the composite null hypothesis is considered in Koning (1993, Ch. 4), where results parallelling those in Koning (1992) are obtained. As in the simple null hypothesis situation, these results may be used in combination with (15) and Lemma 2 to verify the conditions of Theorem 1.

\section{Proofs}

In this section the proofs of Lemma 1, Theorem 1, (15) and Lemma 2 are detailed.

Proof of Lemma 1. Assume that $s_{n}$ remains bounded by $B$. If

$$
\alpha<\exp \left\{-a_{i}\left[C_{i}(\varepsilon, \delta) B\right]^{2}\right\}=: \alpha(\varepsilon, \delta),
$$

then (10) holds for every $\theta \in \Theta-\Theta_{0}$.

Secondly, let us suppose that $\inf _{\theta \in A(\alpha, \varepsilon, \delta)} b_{i}(\theta)=0$ for some $\alpha, \varepsilon, \delta>0$. Let $\left\{\theta_{j}\right\}_{j=1}^{\infty}$ be a sequence such that $\theta_{j} \in A(\alpha, \varepsilon, \delta)$ and $\lim _{j \rightarrow \infty} b_{i}\left(\theta_{j}\right)=0$. Let $n_{j}$ be the smallest integer greater than or equal to

$$
\frac{-\log \alpha}{a_{i}\left\{b_{i}\left(\theta_{j}\right)\right\}^{2}}
$$


Then $\lim _{j \rightarrow \infty} n_{j}=\infty$ and (10) yields

$$
s_{n_{j}}<\frac{n_{j}^{1 / 2} b_{i}\left(\theta_{j}\right)}{C_{i}(\varepsilon, \delta)}
$$

and hence

$$
\liminf _{n \rightarrow \infty} s_{n} \leqslant \frac{1}{C_{i}(\varepsilon, \delta)}\left(\frac{-\log \alpha}{a_{i}}\right)^{1 / 2}<\infty .
$$

Proof of Theorem 1. In view of (12) and (13) choose $\varepsilon_{0}>0, \alpha_{0}>0$ and $0<\xi_{0} \leqslant \frac{1}{2}$ such that

$$
\frac{n_{i}(\alpha, \varepsilon)}{\left[\frac{-2 \log \alpha}{a_{i}\left\{b_{i}(\theta)\right\}^{2}}\right]} \leqslant 1-\xi_{0} \text { and } \inf _{\theta_{0} \in \Theta_{0}^{*}} d\left(\theta, \theta_{0}\right) \leqslant \varepsilon_{i}^{*}
$$

for $i=1,2$, every $0<\alpha<\alpha_{0}, 0<\varepsilon<\varepsilon_{0}$ and $\theta \in A^{*}(\alpha, \varepsilon, \delta)$.

Choose $0<\varepsilon<\varepsilon_{0}$ so as to satisfy $(1-\varepsilon)(1+\varepsilon)^{-3}>1-\xi_{0}$. By Definition 2(b) there exists $t_{0}>0$ such that for every $t \geqslant t_{0}$

$$
(1-\varepsilon) a_{i} t^{2} / 2 \leqslant-\log \left(1-G_{i}(t)\right) \leqslant(1+\varepsilon) a_{i} t^{2} / 2 .
$$

Let $0<\alpha_{1}<\alpha_{0}$ satisfy

$$
\alpha_{1} \leqslant 1-G_{i}\left(t_{0}\right)
$$

(note that $\alpha_{1}$ depends on $\varepsilon$ ). Since for every $0<\alpha<\alpha_{1}$ we have $t_{0} \leqslant \underline{q}_{i}(\alpha)$, we obtain for all $t>\underline{q}_{i}(\alpha)$

$$
-\log \alpha \leqslant-\log \left(1-G_{i}(t)\right) \leqslant(1+\varepsilon) a_{i} t^{2} / 2,
$$

and hence for all $0<\alpha<\alpha_{1}$

$$
-\log \alpha \leqslant(1+\varepsilon) a_{i}\left\{\underline{q}_{i}(\alpha)\right\}^{2} / 2 \text {. }
$$

Similarly, we obtain

$$
-\log \alpha \geqslant(1-\varepsilon) a_{i}\left\{\bar{q}_{i}(\alpha)\right\}^{2} / 2 .
$$

Together with $\underline{q}_{i}(\alpha) \leqslant \bar{q}_{i}(\alpha)$, the inequalities (22) and (23) show that $\underline{q}_{i}(\alpha)$ and $\bar{q}_{i}(\alpha)$ are close to each other.

To derive an upper bound for $\mathrm{N}_{i}(\alpha, \beta, \theta)$, consider $\theta \in A^{*}(\alpha, \varepsilon, \delta)$, and let

$$
n>\frac{(-2 \log \alpha)(1+\varepsilon)}{a_{i}\left\{b_{i}(\theta)\right\}^{2}(1-\varepsilon)^{3}} .
$$

Then by (21)

$$
n \geqslant n_{i}(\alpha, \varepsilon)
$$

and moreover [cf. (23)]

$$
n^{1 / 2} b_{i}(\theta)(1-\varepsilon)>\left\{\frac{(-2 \log \alpha)(1+\varepsilon)}{a_{i}(1-\varepsilon)}\right\}^{1 / 2} \geqslant \bar{q}_{i}(\alpha)(1+\varepsilon)^{1 / 2}
$$


This implies [cf. (5)]

$$
\sup _{\theta_{0} \in \Theta_{0}} P_{\theta_{0}}\left(T_{i n} \geqslant n^{1 / 2} b_{i}(\theta)(1-\varepsilon)\right)<\alpha,
$$

and therefore the power at $\theta$ of the size $\alpha$ test based on $\left\{T_{i n}\right\}_{n=1}^{\infty}$ is at least

$$
P_{\theta}\left(T_{i n}>n^{1 / 2} b_{i}(\theta)(1-\varepsilon)\right) \text {. }
$$

By definition of $A(\alpha, \varepsilon, \delta)$ [cf. (10)], we have

$$
n^{1 / 2} b_{i}(\theta)>C_{i}(\varepsilon, \delta) s_{n}
$$

(8) holds, and thus

$$
P_{\theta}\left(T_{i n}>n^{1 / 2} b_{i}(\theta)(1-\varepsilon)\right)>1-\delta \geqslant \beta .
$$

So for all $\mathrm{n}$ satisfying (24) the power of the size $\alpha$ test based on $\left\{T_{i n}\right\}_{n=1}^{\infty}$ is greater than $\beta$, and hence

$$
\mathrm{N}_{i}(\alpha, \beta, \theta) \leqslant \frac{(-2 \log \alpha)(1+\varepsilon)}{a_{i}\left\{b_{i}(\theta)\right\}^{2}(1-\varepsilon)^{3}}
$$

for the chosen $0<\varepsilon<\varepsilon_{0}$, every $0<\alpha<\alpha_{i}$, and every $\theta \in A^{*}(\alpha, \varepsilon, \delta)$.

Remains to find a lower bound for $\mathrm{N}_{i}(\alpha, \beta, \theta)$. Again, let $\theta \in A^{*}(\alpha, \varepsilon, \delta)$. Since $(1-\varepsilon)(1+\varepsilon)^{-3}>1-\xi_{0} \geqslant \frac{1}{2}$, we may choose $n$ so as to satisfy

$$
\frac{-\log \alpha}{a_{i}\left\{b_{i}(\theta)\right\}^{2}} \leqslant \frac{-2 \log \alpha}{a_{i}\left\{b_{i}(\theta)\right\}^{2}}\left(1-\xi_{0}\right) \leqslant n<\frac{(-2 \log \alpha)(1-\varepsilon)}{a_{i}\left\{b_{i}(\theta)\right\}^{2}(1+\varepsilon)^{3}}
$$

Then by (21)

$$
n \geqslant n_{i}(\alpha, \varepsilon)
$$

and moreover [cf. (22)]

$$
n^{1 / 2} b_{i}(\theta)(1+\varepsilon)<\left\{\frac{(-2 \log \alpha)(1-\varepsilon)}{a_{i}(1+\varepsilon)}\right\}^{1 / 2} \leqslant \underline{q}_{i}(\alpha)(1-\varepsilon)^{1 / 2}
$$

This implies [cf. (6)]

$$
\sup _{\theta_{0} \in \Theta_{0}} P_{\theta_{0}}\left(T_{i n}>n^{1 / 2} b_{i}(\theta)(1+\varepsilon)\right)>\alpha,
$$

and therefore the power at $\theta$ of the size $\alpha$ test based on $\left\{T_{i n}\right\}_{n=1}^{\infty}$ is at most

$$
P_{\theta}\left(T_{\text {in }}>n^{1 / 2} b_{i}(\theta)(1+\varepsilon)\right) .
$$

Since

$$
n \geqslant \frac{-\log \alpha}{a_{i}\left\{b_{i}(\theta)\right\}^{2}}
$$

it follows by $(10)$ that $n^{1 / 2} b_{i}(\theta)>C_{i}(\varepsilon, \delta) s_{n}$, and thus in view of (8)

$$
P_{\theta}\left(T_{i n} \geqslant n^{1 / 2} b_{i}(\theta)(1+\varepsilon)\right)<\delta \leqslant \beta .
$$


Hence we obtain

$$
\mathrm{N}_{i}(\alpha, \beta, \theta) \geqslant \frac{(-2 \log \alpha)(1-\varepsilon)}{a_{i}\left\{b_{i}(\theta)\right\}^{2}(1+\varepsilon)^{3}}
$$

for the chosen $0<\varepsilon<\varepsilon_{0}$, every $0<\alpha<\alpha_{1}$, and every $\theta \in A^{*}(\alpha, \varepsilon, \delta)$. Combination of (25) and (26) yields the theorem.

Proof of (15). Since $s_{n}=\mathcal{O}\left(n^{\rho}\right)$, we may choose $C_{i}^{*}(\varepsilon, \delta)$ so as to satisfy $C_{i}(\varepsilon, \delta) s_{n} \leqslant C_{i}^{*}(\varepsilon, \delta) n^{\rho}$ for every $n$ and every $\varepsilon, \delta>0$. Let $\theta$ satisfy

$$
C_{i}^{*}(\varepsilon, \delta) \leqslant\left(\frac{-\log \alpha}{a_{i}}\right)^{1 / 2-\rho}\left\{b_{i}(\theta)\right\}^{2 \rho},
$$

and let $n \geqslant(-\log \alpha) / a_{i}\left\{b_{i}(\theta)\right\}^{2}$. It follows that

$$
n^{1 / 2-\rho} b_{i}(\theta) \geqslant\left(\frac{-\log \alpha}{a_{i}\left\{b_{i}(\theta)\right\}^{2}}\right)^{1 / 2-\rho} b_{i}(\theta) \geqslant\left(\frac{-\log \alpha}{a_{i}}\right)^{1 / 2-\rho}\left\{b_{i}(\theta)\right\}^{2 \rho} \geqslant C_{i}^{*}(\varepsilon, \delta),
$$

and hence

$$
n^{1 / 2} b_{i}(\theta) \geqslant n^{1 / 2-\rho} b_{i}(\theta) n^{\rho} \geqslant C_{i}^{*}(\varepsilon, \delta) n^{\rho} \geqslant C_{i}(\varepsilon, \delta) s_{n} .
$$

thus completing the proof of (15).

Proof of Lemma 2. From (18) we have for any $\alpha$ and $n$

$$
\sup _{\theta_{0} \in \Theta_{0}} P_{\theta_{0}}\left(\left|T_{i n}-T_{i}\right|>n^{-\gamma}\left(k_{n}+\frac{2}{\lambda}|\log \alpha|\right)^{\kappa}\right) \leqslant \alpha^{2} .
$$

Let

$$
n \geqslant c^{-1}|2 \log \alpha|^{(\kappa-1 / 2) / \gamma}
$$

for some $c>0$, and let $\alpha$ tend to zero. Since $k_{n}=\mathcal{O}\left(n^{\gamma / \kappa}\right)$ and

$$
\lim _{\alpha \rightarrow 0} \frac{\bar{q}_{i}(\alpha)}{|2 \log \alpha|^{1 / 2}}=a_{i}^{-1 / 2}
$$

in view of (22) and (23), we obtain

$$
\liminf _{\alpha \rightarrow 0} \frac{\bar{q}_{i}(\alpha)(1+\varepsilon)^{1 / 2}-n^{-\gamma}\left(k_{n}+\frac{2}{\lambda}|\log \alpha|\right)^{\kappa}}{|2 \log \alpha|^{1 / 2}} \geqslant a_{i}^{-1 / 2}(1+\varepsilon)^{1 / 2}-c^{\gamma} \lambda^{-\kappa} .
$$

Application of (2) yields

$$
\begin{aligned}
& \liminf _{\alpha \rightarrow 0} \frac{\log \left(1-G_{i}\left(\bar{q}_{i}(\alpha)(1+\varepsilon)^{1 / 2}-n^{-\gamma}\left(k_{n}+\frac{2}{\lambda}|\log \alpha|\right)^{\kappa}\right)\right)}{\log \alpha} \\
& \geqslant\left\{(1+\varepsilon)^{1 / 2}-a_{i}^{1 / 2} c^{\gamma} \lambda^{-\kappa}\right\}^{2},
\end{aligned}
$$


which is larger than 1 by taking $c=c(\varepsilon)$ sufficiently small. Combining (27), (29) and

$$
\begin{aligned}
1-\inf _{\theta_{0} \in \Theta_{0}} P_{\theta_{0}}\left(T_{i n} \leqslant \bar{q}_{i}(\alpha)(1+\varepsilon)^{1 / 2}\right) \leqslant & 1-G_{i}\left(\bar{q}_{i}(\alpha)(1+\varepsilon)^{1 / 2}-n^{-\gamma}\left(k_{n}+\frac{2}{\lambda} \mid \log \alpha\right)^{\kappa}\right) \\
& +\sup _{\theta_{0} \in \Theta_{0}} P_{\theta_{0}}\left(\left|T_{i n}-T_{i}\right|>n^{-\gamma}\left(k_{n}+\frac{2}{\lambda}|\log \alpha|\right)^{\kappa}\right)
\end{aligned}
$$

leads to

$$
\lim _{\alpha \rightarrow 0} \frac{1-\inf _{\theta_{0} \in \Theta_{0}} P_{\theta_{0}}\left(T_{i n} \leqslant \bar{q}_{i}(\alpha)(1+\varepsilon)^{1 / 2}\right)}{\alpha}=0,
$$

implying that $\bar{n}_{i}(\alpha, \varepsilon)$ does not exceed the right-hand side of $(28)$ for $\alpha$ sufficiently small. Similarly, an upper bound for $\underline{n}_{i}(\alpha, \varepsilon)$ is derived, and (13) follows.

\section{References}

Aki, S. (1986), Some test statistics based on the martingale term of the empirical distribution function, Ann. Institute Statist. Math. 38A, $1-21$

Andersen, P.K., O. Borgan, R.D. Gill and N. Keiding (1993), Statistical Methods based on Counting Processes (Springer, New York). Bahadur, R.R. (1960), Stochastic comparisons of tests, Ann. Math. Statist. 31, 276-295.

Breslow, N.E. (1975), Analysis of survival data under proportional hazards model, Internat. Statist. Rev. 43, 45-58.

Csörgő, M. and L. Horváth (1993), Weighted Approximations in Probability and Statistics (Wiley, New York).

Harrington, D.P. and T.R. Fleming (1982), A class of rank test procedures for censored survival data, Biometrika 69, 553-566.

Kallenberg, W.C.M. (1983), Intermediate efficiency, theory and examples, Ann. Statist. 11, 170-182.

Komlós, J., P. Major and G. Tusnády (1975), An approximation of partial sums of independent R.V.'s and the sample F.I. Z, Wahrsch. verw. Gebiete 34, 33-58.

Koning, A.J. (1992), Approximation of stochastic integrals with applications to goodness-of-fit tests, Ann. Statist. $20,428-454$.

Koning, A.J. (1993), Stochastic Integrals and Goodness-of-fit Tests, CWI Tract 98. CWI, Amsterdam.

Koning, A.J. (1994a), Approximation of the basic martingale, Ann. Statist. 22, 565-579.

Koning, A.J. (1994b), KMT-type inequalities and goodness-of-fit tests, Statistica Neerlandica 48, 117-132.

Marcus, M.B and J. Zinn (1984), The bounded law of the iterated logarithm for the weighted empirical distribution process in the non-i.i.d. case, Ann. Probab. 12, 335-360.

Pyke, R. and G.R. Shorack (1968), Weak convergence of a two-sample empirical process and a new approach to Chernoff-Savage theorems, Ann. Math. Statist. 39, 755-771.

Rothe, G. (1981), Some properties of the asymptotic relative Pitman efficiency, Ann. Statist. 9, 663-669.

Wieand, H.S. (1976), A condition under which the Pitman and Bahadur approaches to efficiency coincide, Ann. Statist. 4, 1003-1011. 